\title{
LRRK2 is involved in the pathogenesis of system lupus erythematosus through promoting pathogenic antibody production
}

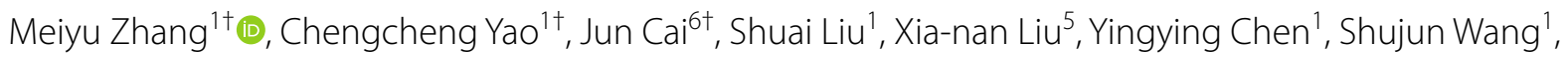
Ping Ji ${ }^{1}$, Meng Pan ${ }^{2}$, Zizhen Kang ${ }^{3,4^{*}}$ and Ying Wang ${ }^{1^{*}}$

\begin{abstract}
Background: Systemic lupus erythematosus (SLE) is a prototypic autoimmune disease characterized by the presence of pathogenic autoantibodies associated with polyclonal B cell hyperreactivity. Previous study reported that autophagy-related gene Leucine-rich repeat kinase 2 (LRRK2) was likely a susceptible gene for SLE. However, the pathogenic function of LRRK2 in SLE is undefined.
\end{abstract}

Methods: Using quantitative PCR, we compared the expression levels of LRRK2 in B cells between SLE patients and healthy controls. The expression levels of $L R R K 2$ in in vitro induced CD19hi B cells and naïve B cells were compared as well based on RNA-seq assay. A pristane-induced lupus-like mouse model was used to explore the effects of LRRK2 on the development of SLE. IgG level, B cell subsets in the spleens and bone marrows and pathological features in the kidneys were compared between wildtype (WT) and Lrrk2 ${ }^{-/}$littermates.

Results: It was obvious that $L R R K 2$ expression was dramatically up-regulated in primary B cells from SLE patients compared to those from healthy controls, as well as in activated CD19 hi B cells. More significantly, LRRK2 expression in B cells was positively correlated with system lupus erythematosus disease activity index (SLEDAI), an indicator for disease severity, and serum lgG levels in SLE patients. Negative correlations were observed between LRRK2 expression and serum C3 or C4 levels, two clinical features associated with SLE-related nephritis. LRRK2 deficiency reduced the death rate of pristane treated mice. Decreased levels of total lgG and autoantibody were detected in the serum with less deposition of immune complexes and attenuated pathological symptoms in the kidneys of Lrrk2 ${ }^{-1-}$ mice. Consistent with the reduction in IgG production, the percentages of germinal center B cells and plasma cells decreased significantly as well with LRRK2 deficiency.

Conclusions: Our study demonstrates that LRRK2 expression is upregulated in B cells from SLE patients with strong correlations to disease severity. LRRK2 deficiency largely attenuates the pathogenic progress of lupus-like features in pristane-induced mice. This is probably achieved through affecting B cell terminal differentiation and subsequent immunoglobulin production.

\footnotetext{
*Correspondence: zizhenkang@gmail.com; ywang@sibs.ac.cn

${ }^{\dagger}$ Meiyu Zhang, Chengcheng Yao and Jun Cai contributed equally to this work

1 Shanghai Institute of Immunology, Department of Immunology and Microbiology, Shanghai Jiao Tong University School of Medicine, Shanghai 200025, China

${ }^{3}$ Department of Inflammation and Immunity, Cleveland Clinic, Cleveland, $\mathrm{OH}$ 44195, USA

Full list of author information is available at the end of the article
}

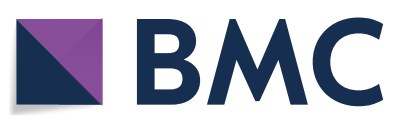

(c) The Author(s) 2019. This article is distributed under the terms of the Creative Commons Attribution 4.0 International License (http://creativecommons.org/licenses/by/4.0/), which permits unrestricted use, distribution, and reproduction in any medium, provided you give appropriate credit to the original author(s) and the source, provide a link to the Creative Commons license, and indicate if changes were made. The Creative Commons Public Domain Dedication waiver (http://creativecommons.org/ publicdomain/zero/1.0/) applies to the data made available in this article, unless otherwise stated. 
Keywords: System lupus erythematosus, Leucine-rich repeat kinase 2, Lupus-like mouse model, B cell differentiation, Antibody production

\section{Background}

Systemic lupus erythematosus (SLE) is a severe heterogeneous systemic autoimmune disease characterized by the production of autoantibodies against certain self-antigens. The formation and deposition of immune complexes (ICs) in various tissues and organs result in local inflammatory responses and severe tissue destruction including the brain, blood, heart and kidney [1]. Polyclonal B cell hyperreactivity is considered to be one of the main immuno-pathological properties in SLE [2]. Successful application of Rituximab, a chimeric mousehuman monoclonal antibody against B cell-specific antigen CD20, in clinic highlights the role of B cells in the pathogenesis of SLE $[3,4]$.

It is demonstrated that B cell activation in SLE is comprehensively regulated by both intrinsically genetic and extrinsically environmental factors [5]. Multiple cytokines related to $B$ cell activation, such as IFN- $\alpha$ mainly derived from plasmacytoid dendritic cells (pDCs) and Th2 cytokines such as IL-4, IL-5 et al., are elevated in SLE patients [6, 7]. Most intrinsic factors are implied from the viewpoint of genetics based on genomewide associated analysis (GWAS) [8]. Among them, an autophagy-related gene encoding leucine-rich repeat kinase 2 (LRRK2) was recently identified [9]. LRRK2 contains 2527 amino acid residues with several functional domains, including a leucine-rich repeats (LRR) domain, a Ras of complex proteins (ROC) domain, a C-terminal of ROC (COR) domain, a kinase domain and a WD40repeat domain [10]. LRRK2 is reported to be highly expressed in certain immune cells, such as B cells, monocytes, macrophages, and microglia whereas less in T cells [11]. It has recently been reported to be associated with multiple diseases including Parkinson's disease $[12,13]$, leprosy [14], inflammatory bowel disease (IBD) $[15,16]$ and cancers [17].

Emerging data suggest that LRRK2 plays critical roles in immune modulation of macrophages, which is supported by the investigations on the resistance of LRRK2 to intracellular pathogen infection, including Listeria monocytogenes [18] and Mycobacterial tuberculosis [19]. Our recent study also suggested that LRRK2 was critical for NLRC4 inflammasome activation in macrophages, which was indispensable for host defense against Salmonella infection [20]. In addition to the involvement of LRRK2 in innate immunity, the roles of LRRK2 in B cells were firstly proposed due to its high expression in peripheral B cells in an age-dependent manner [21].
Considering its susceptibility to SLE and high expression in B cells, whether LRRK2 functions in the pathogenesis of SLE is worthy of investigation.

In this study, we found that LRRK2 expression was dramatically increased in B cells from SLE patients compared to that from healthy controls (HCs). Of note, LRRK2 expression in B cells was positively correlated with disease severity and the levels of serum IgG in SLE patients. Furthermore, we demonstrated that LRRK2 promoted B cell terminal differentiation, humoral immune response and consequently lupus-like syndrome in a pristaneinduced mouse model, thus implicating LRRK2 as a novel target in SLE therapy.

\section{Methods}

Human subjects

SLE patients $(n=22)$ enrolled in this study were from Ruijin Hospital affiliated to Shanghai Jiao Tong University School of Medicine (Shanghai, China). All SLE patients fulfilled the American Rheumatism Association Criteria for the diagnosis of SLE. The study was approved by the Ethic Committee of Ruijin Hospital affiliated to Shanghai Jiao Tong University School of Medicine. All experiments were performed according to the principles of the Declaration of Helsinki. Informed consent forms were assigned individually. HCs $(n=31)$ were volunteer donors undergoing annual physical examination. SLE patients and HCs were both gender and age matched (Table 1).

\section{Cell isolation}

Whole blood was collected in heparin lithium-treated tubes. Peripheral blood mononuclear cells (PBMCs) were isolated by density gradient centrifugation using Lymphoprep $^{\mathrm{TM}}$ reagent (Axis-shield, Norway). CD4 ${ }^{+} \mathrm{T}$ cells from SLE patients or HCs were isolated by using a human CD4 MicroBeads kit (Miltenyi Biotec, Bergisch Gladbach, Germany) according to the manufacturer's instructions. Briefly, PBMCs were incubated in PBS supplemented with $0.5 \%$ bovine serum albumin (BSA) containing CD4 MicroBeads. Cell suspension was applied on an MS separation column placing in the magnetic field. Unlabeled cells were collected for subsequent B cell purification. The MS column was removed from the magnetic field and $\mathrm{CD} 4^{+} \mathrm{T}$ cells were eluted by rinsing the column with PBS. B cells were further purified with an EasySep ${ }^{\text {TM }}$ Human Naïve B Cell Enrichment Kit (STEMCELL Technologies, Canada) accordingly. Briefly, the elution cell fraction after $\mathrm{CD} 4^{+} \mathrm{T}$ cell purification was incubated 
Table 1 Demographic and clinical characteristics of SLE and healthy controls

\begin{tabular}{llc}
\hline Characteristics & Healthy controls $(\mathbf{n = 3 1 )}$ & SLE patients $(\mathbf{n}=\mathbf{2 2})$ \\
\hline Age (years) & $42.29 \pm 11.38(24-62)$ & $36.29 \pm 13.79(13-71)$ \\
Gender (female/male) & $27 / 4$ & $20 / 2$ \\
Disease duration (years) & - & $6.5 \pm 6.07$ \\
Renal diseases n (\%) & - & $15(68.1)$ \\
Neurological disorder $n(\%)$ & - & $3(13.6)$ \\
Thrombocytopenia n (\%) & - & $5(22.7)$ \\
Anti-ds-DNA antibody n (\%) & - & $15(68.1)$ \\
IgG (mg/dL) & - & $1277 \pm 388$ \\
IgM (mg/dL) & - & $81 \pm 59$ \\
IgA (mg/dL) & - & $300 \pm 128$ \\
SLEDAl & & $6.27 \pm 5.22(2-20)$ \\
\hline
\end{tabular}

with enrichment cocktail for $10 \mathrm{~min}$ at room temperature (RT). Magnetic particles were added in the mixture for $5 \mathrm{~min}$ incubation at RT. The tubes were placed in the magnet at RT for $5 \mathrm{~min}$. The enriched cell suspension was then carefully transferred into a new tube. The purity of CD4 ${ }^{+} \mathrm{T}$ cells and $\mathrm{B}$ cells was determined by flow cytometry. Cells with the purity $>95 \%$ were used for further experiments.

RNA extraction, reverse transcription, and quantitative PCR Total RNA was extracted from PBMCs, purified CD4 ${ }^{+}$ $\mathrm{T}$ cells or $\mathrm{B}$ cells by using the TRIzol reagent (Invitrogen, Carlsbad, CA, USA). NanoDrop spectrophotometer (Thermo Scientific, Waltham, MA, USA) was applied for RNA concentration determination. cDNA was synthesized by using the PrimeScriptTM RT reagent Kit (TaKaRa, Kusatsu, Japan). Semi-quantitative polymerase chain reaction (qPCR) was carried out with the SYBR PremixEx TaqTM II kit (TaKaRa) in a ViiA 7 Real-Time PCR System (Applied Biosystems, Carlsbad, CA, USA). The primers specific to $L R R K 2$ were as forward: $5^{\prime}$-GAGCAC GCCTCCAAGTTATTT- $3^{\prime}$ and reverse: 5'-ACTGGC ATTATGAACTGTTAGCA-3'. House-keeping gene $G A P D H$ was used as an internal control (primers: forward: 5'-GGAGCGAGATCCCTCCAAAAT-3'; reverse: 5'-GGCTGTTGTCATACTTCTCATGG-3'). The expression level of $L R R K 2$ was calculated based on cycle threshold $(\mathrm{Ct})$ values of target gene and GAPDH. The relative expression level of $L R R K 2=2^{\wedge}\left(\mathrm{Ct}_{G A P D H}-\mathrm{Ct}_{L R R K 2}\right)$.

\section{Mice}

Lrrk2 ${ }^{-/-}$mice (C57BL/6 N-Lrrk2 $2^{\text {tm 1.Miff/J }}$; approved by the Michael J. Fox Foundation for Parkinson's research; JAX stock 016121) were purchased from the Jackson Laboratory (USA) and backcrossed onto a C57BL/6 background for at least six generations. All mice were bred and maintained in individually ventilated cages under specific pathogen-free conditions in the accredited animal facility of Shanghai Jiao Tong University School of Medicine (Shanghai, China). Age- and gender-matched wildtype (WT) mice at 8-10 weeks' old were used as littermate controls in all experiments. To ameliorate the suffering of mice, all mice were sacrificed through $\mathrm{CO}_{2}$ inhalation in the studies.

\section{Preparation of lupus-like mice by pristane injection}

8-10-week-old age- and gender-matched WT and Lrrk $2^{-1-}$ female mice were administered intraperitoneally with $500 \mu \mathrm{L}$ pristane $(2,6,10,14$-tetramethylpentadecane) (Sigma-Aldrich, St Louis, MO, USA). 50-100 $\mu \mathrm{L}$ blood was collected from eye veins with an interval of 1 month for serum preparation. Mice were sacrificed at 9 months after pristane injection. Immunological and pathological characters were assessed.

\section{Flow cytometry}

The spleens were collected from WT and Lrrk2-1pristane-treated mice at 9 months. Splenocytes were prepared by density gradient centrifugation using Lymphoprep $^{\mathrm{TM}}$ reagent (Axis-shield). For flow cytometry, splenocytes were washed once with FACS buffer $(1 \times$ PBS supplemented with $2 \%$ fetal bovine serum) (Millipore, Billerica, MA, USA) and stained with antibodies against surface molecules, including anti-CD45R/B220-Percpcy5.5, anti-CD93-phycoerythrin/cyanin (PE-Cy7), antiIgM-allophyco-cyanin (APC-Cy7), anti-CD38-APC, anti-CD138-APC, anti-GL7-fluorescein isothiocyanate (FITC) (all from Biolegend, San Diego, CA, USA) and anti-CD23-PE (Thermo Scientific) antibodies diluted in FACS buffer. Cells were incubated at $4{ }^{\circ} \mathrm{C}$ for $30 \mathrm{~min}$, and collected on an LSR Fortessa (BD Biosciences, San Diego, CA, USA). Data analysis was carried out with FlowJo 7.6 software (Tree Star, Ashland, OR, USA). 


\section{Enzyme linked immune sorbent assay (ELISA)}

Blood was kept at RT for $2 \mathrm{~h}$ and centrifuged at $5500 \mathrm{rpm}$ for $10 \mathrm{~min}$. Sera were collected and stored at $-80{ }^{\circ} \mathrm{C}$ for ELISA assay. For determination of total IgG and IgM in the serum, 96-well polystyrene plates (Corning, NY, USA) were coated with $2 \mu \mathrm{g} / \mathrm{mL}$ goat anti-mouse $\operatorname{IgG}(\mathrm{H}+\mathrm{L})$ or goat anti-mouse IgM (SouthernBiotech, Birmingham, AL, USA) antibodies diluted in bicarbonate/carbonate coating buffer $\left(15 \mathrm{mM} \mathrm{Na} \mathrm{CO}_{3}\right.$ and $35 \mathrm{mM} \mathrm{NaHCO}, \mathrm{pH}$ 9.6), and incubated at $4{ }^{\circ} \mathrm{C}$ overnight. After washing 3 times with PBST (PBS containing 0.05\% Tween-20) (Sangon Biotech, Shanghai, China), the wells were blocked with $200 \mu \mathrm{L} /$ well PBS containing $5 \%$ skimmed milk powder at RT for $2 \mathrm{~h}$. Mouse sera were serially diluted in PBST containing 3\% skimmed milk powder. $100 \mu \mathrm{L}$ diluted serum was added to each well and the plates were incubated at RT for $2 \mathrm{~h}$. After washing 3 times with PBST, the wells were incubated with horseradish peroxidase (HRP)-conjugated goat antimouse antibody for $\operatorname{IgG}(\mathrm{H}+\mathrm{L})$ and $\mathrm{HRP}$-conjugated goat anti-mouse antibody for IgM (SouthernBiotech) working solution (1:4000 diluted in PBST containing 3\% skimmed milk powder, $100 \mu \mathrm{L} /$ well) at RT for $2 \mathrm{~h}$. Tetramethylbenzidine (TMB) (BD Bioscience) solution $(100 \mu \mathrm{L} /$ well) was added, and the plates were incubated at RT in the dark for $15 \mathrm{~min}$. The reaction was stopped by adding $1 \mathrm{M} \mathrm{H}_{2} \mathrm{SO}_{4}(50 \mu \mathrm{L} /$ well $)$. The absorbance at $450 \mathrm{~nm}$ was detected within 5 min by PowerWaveXS2 microplate spectrophotometer (BioTek, Burlington, VT, USA).

For the detection of anti-ribonucleoprotein (RNP) and anti-double strand DNA (dsDNA) antibodies, 96-well ELISA plates (Corning) were coated with $0.3 \mu \mathrm{g} / \mathrm{mL}$ (100 $\mu \mathrm{L} /$ well) U1-SnRNP (for anti-RNP autoantibody) (Diarect, Freiburg, Germany) and $3 \mu \mathrm{g} / \mathrm{mL}(100 \mu \mathrm{L} /$ well $)$ dsDNA (for anti-dsDNA Ab) (Diarect) diluted in coating buffer and incubated at $4{ }^{\circ} \mathrm{C}$ overnight. After washing 3 times with PBST, the wells were blocked with $200 \mu \mathrm{L} /$ well blocking buffer at RT for $2 \mathrm{~h} .100 \mu \mathrm{L} /$ well diluted serum was added to the wells and the plates were incubated at RT for $2 \mathrm{~h}$. After washing 3 times with PBST, HRP-conjugated goat anti-mouse antibody for $\operatorname{IgG}(\mathrm{H}+\mathrm{L})$ (SouthernBiotech) working solution (1:4000 diluted in PBST containing $3 \%$ skimmed milk powder, $100 \mu \mathrm{L} /$ well) was added and incubated at RT for $2 \mathrm{~h}$. TMB (BD Bioscience) solution $(100 \mu \mathrm{L} /$ well) was added later, and the plates were incubated at RT in the dark for $15 \mathrm{~min}$. The reaction was stopped by adding $1 \mathrm{M} \mathrm{H}_{2} \mathrm{SO}_{4}(50 \mu \mathrm{L} /$ well $)$. The absorbance at $450 \mathrm{~nm}$ was detected within $5 \mathrm{~min}$ by PowerWaveXS2 microplate spectrophotometer (BioTek).

\section{Histology and immunofluorescence assay (IFA)}

Mouse kidneys were fixed in PBS containing 4\% paraformaldehyde at RT and embedded in the paraffin routinely. Paraffin sections $(4 \mu \mathrm{m})$ were stained with hematoxylin and eosin $(\mathrm{H} \& \mathrm{E})$. Sections were scanned with OLYMPUS microscope (Tokyo, Japan) equipped with a $40 \times$ objective. Glomerulonephritis in the kidneys was assessed using a semiquantitative (1-4+) scoring scale based on the number of the glomeruli with endocapillary proliferation and extracapillary proliferation (crescents) as following: no glomeruli $=0 ; 1-25 \%$ of glomeruli $=1 ; 26-50 \%$ of glomeruli $=2 ; 51-75 \%$ of glomer$\mathrm{uli}=3$; and $76-100 \%$ of glomeruli $=4+$. The slides were double-blinded examined by a pathologist. At least five randomly selected images were undergone analysis.

The deposition of IgG in the kidneys was examined by direct IFA with paraffin sections. The slides were stained with fluorochrome-conjugated anti-IgG Ab (Abcam, \#ab6785) with a 1:500 dilution and incubated at $4{ }^{\circ} \mathrm{C}$ for $24 \mathrm{~h}$. The slides were washed with PBS and air-dried. 4,6-diamidino-2-phenylindole (DAPI) FluoromountG (YEASEN, Shanghai, China) was added on the slides before the observation. Fluorescence signal was determined under a TCS SP8 confocal microscope (Leica, Solms, Germany). Data analysis was carried out with ImageJ software (National Institutes of Health, Bethesda, MD, USA). At least five fields were randomly selected for quantification analysis.

\section{Statistical analysis}

Data were presented as mean \pm standard error of means (S.E.M). Statistical analyses were performed by using Graphpad Prism 5.0 software (Graphpad Prism, La Jolla, CA, USA). Statistic difference was determined by unpaired Student $t$ test for the data with gaussian distribution, and by Mann-Whitney test for those with non-gaussian distribution. Unless stated, $\mathrm{p}<0.05$ was considered statistically significant.

\section{Results}

Up-regulation of LRRK2 in B cells from SLE patients as well as in activated $B$ cells

To explore the possible relationship between LRRK2 and SLE pathogenesis, the expression levels of LRRK2 in PBMCs were firstly compared between SLE patients and $\mathrm{HC}$ donors (Table 1). Consistent with the previous study [9], LRRK2 expression in PBMCs was significantly increased in SLE patients when compared to HCs (Fig. 1a). CD4 ${ }^{+} \mathrm{T}$ cells and B cells from SLE patients or HCs were further isolated separately with high purity (Additional file 1: Figure S1) and the expression levels of $L R R K 2$ in cell subsets were determined. When precisely comparing $L R R K 2$ expression in $\mathrm{CD}^{+}{ }^{+} \mathrm{T}$ cells and B cells, it was obvious that B cells from both SLE and HCs groups expressed $L R R K 2$ more dramatically than $C D 4^{+} \mathrm{T}$ cells. 

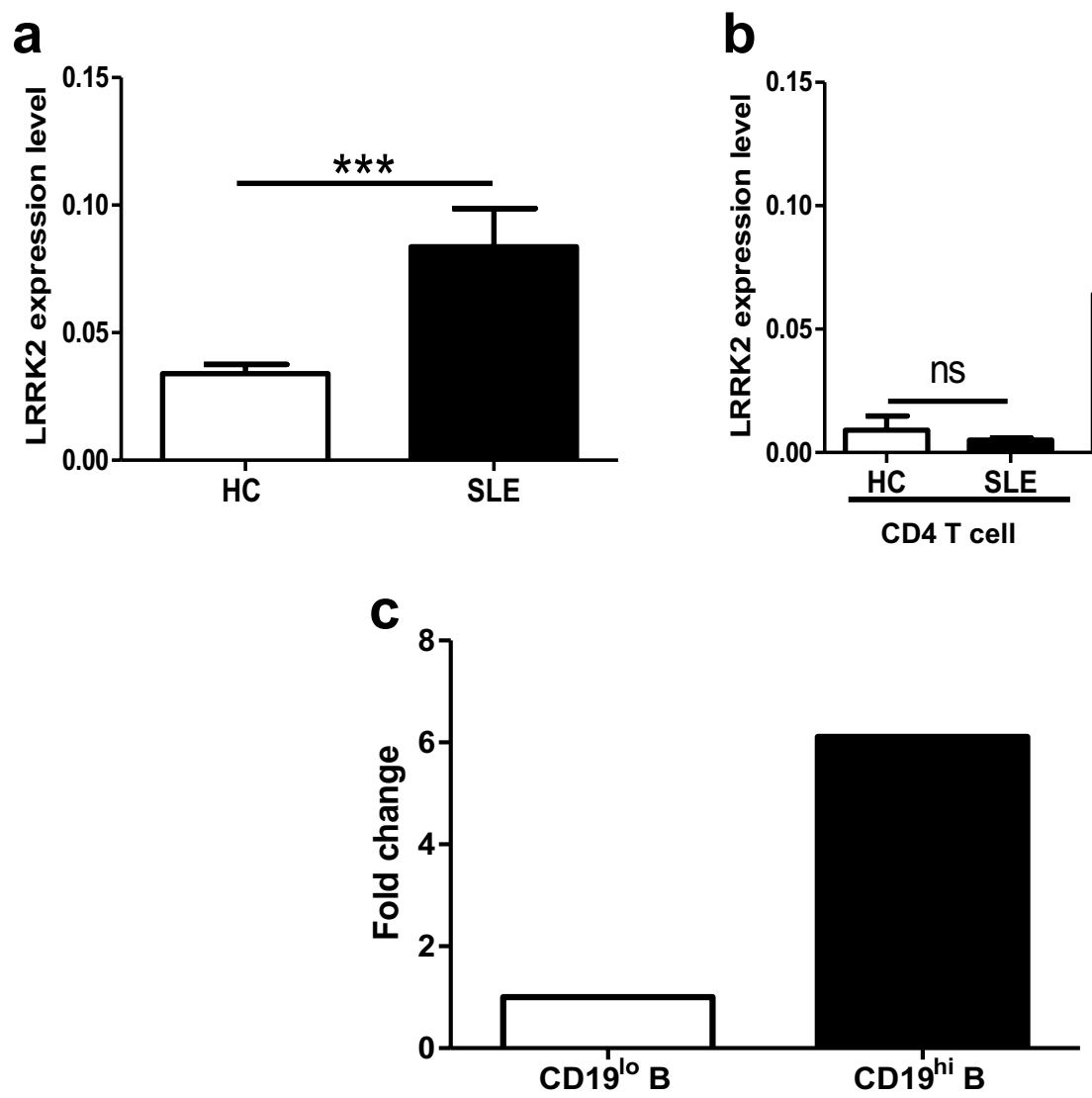

Fig. 1 LRRK2 expression profiles in the peripheral immune cells. Whole blood was collected from SLE patients and healthy volunteer donors. The expression levels of $\angle R R K 2$ in PBMCs (a), CD4 ${ }^{+}$T cells and B cells (b) from SLE patients and healthy donors were determined by RT-qPCR. $\mathbf{c}$ The expression levels of $\angle R R K 2$ in resting $C D 19^{l o} B$ cells and CD19 hi $B$ cells from three healthy donors were calculated based on transcriptome assay data [24]. ${ }^{*} p<0.05 ;{ }^{* * *} p<0.001$

\section{b}

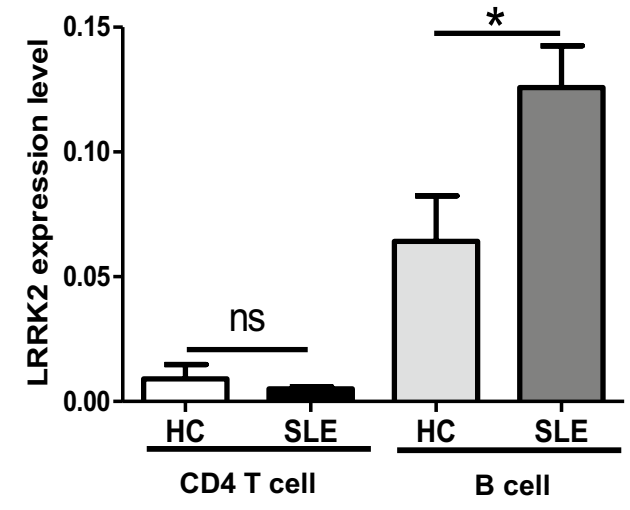

More significantly, the expression of $L R R K 2$ was elevated in B cells from SLE patients than that from HCs whereas was comparable in $\mathrm{CD} 4^{+} \mathrm{T}$ cells from SLE patients and $\mathrm{HC}$ individuals (Fig. 1b).

CD19 ${ }^{\text {hi }}$ B cells were previously reported existing in certain antibody-driven autoimmune diseases, such as common variable immunodeficiency (CVID) [22], SLE [23] and pemphigus [24]. These CD19 ${ }^{\text {hi }} B$ cells exhibit activated phenotypes, and function in promoting antibody production in autoimmune diseases. CD19 ${ }^{\text {hi }} \mathrm{B}$ cells can be induced through co-culturing activated $\mathrm{CD} 4^{+} \mathrm{T}$ cells with homologous resting $\mathrm{B}$ cells $\left(\mathrm{CD} 19^{\mathrm{lo}} \mathrm{B}\right.$ cells) in vitro from healthy donors [24]. Relying on transcriptome analysis of $\mathrm{CD} 19^{\text {hi }} \mathrm{B}$ cells and homologous $\mathrm{CD} 19^{\text {lo }}$ B cells from healthy donors, we compared $L R R K 2$ expression between these two types of $B$ cell populations. It was found that more LRRK2 expression was detectable in CD19hi B cells than in the resting counterparts (Fig. 1c). These results strongly imply that LRRK2 expression is associated with B cell activation and antibody 

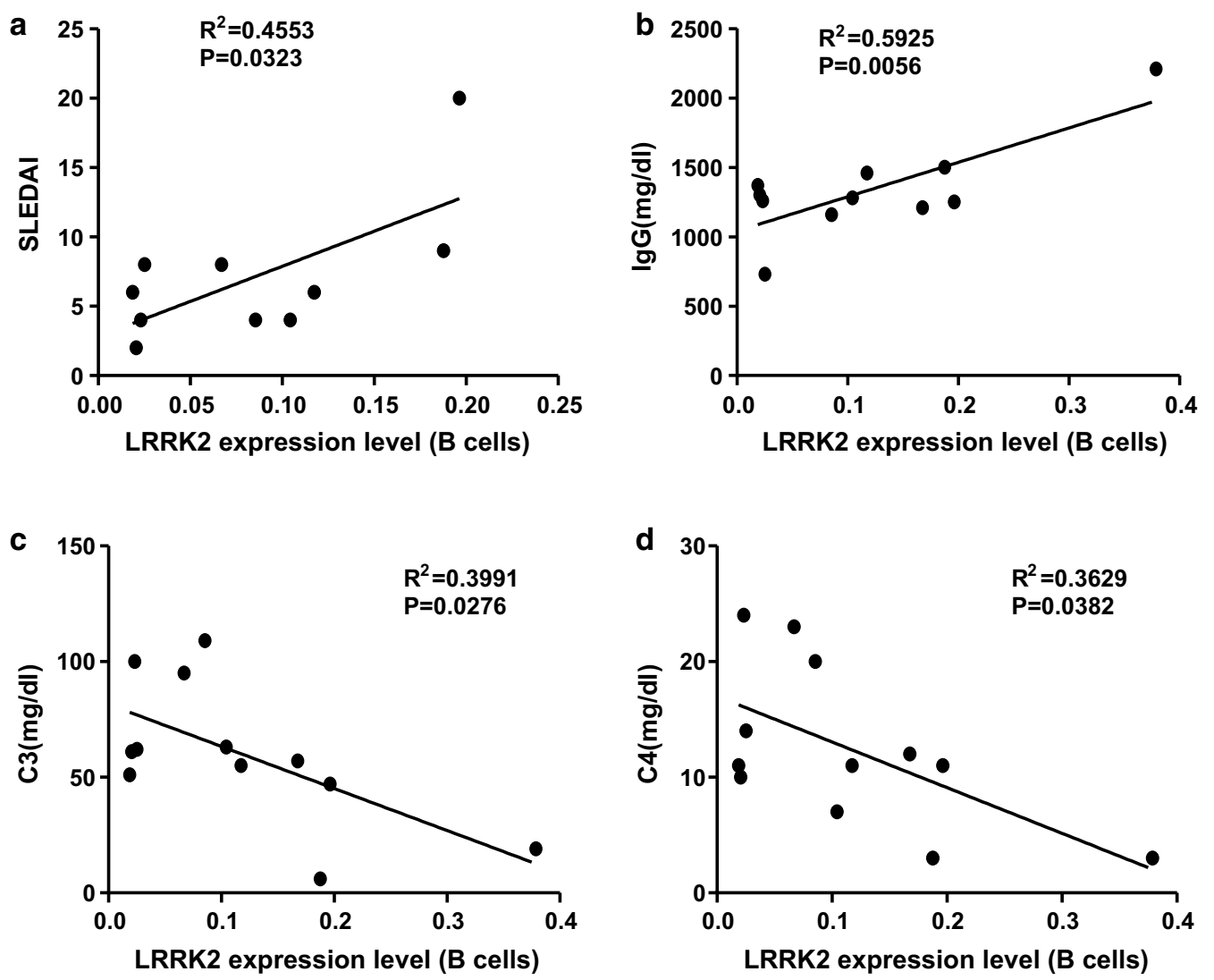

Fig. 2 Correlation analysis between LRRK2 expression in B cells and clinical manifestations of SLE. LRRK2 expression in purified B cells from SLE patients was determined by semi-qPCR with GAPDH as an internal control. Correlations between LRRK2 expression in B cells and SLEDAI (a), IgG level (b), C3 (c) and C4 (d) were determined. The correlation coefficient $r$ and the p value were calculated using the Spearman rank test

the decrease in $\mathrm{C} 3$ and $\mathrm{C} 4$ in the periphery is associated with disease severity of SLE, especially the occurrence of SLE-related nephritis [25]. Contrarily, LRRK2 expression in B cells was negatively correlated with both serum C3 (Fig. 2c) and C4 levels (Fig. 2d). Although not all 22 SLE patients were included in correlation study due to the incomplete clinical examination parameters, the fact that LRRK2 expression in B cells exhibits strong correlations with disease severity of SLE in partial patients implies overwhelming roles of LRRK2 in the pathologies of SLE.

\section{Comparable B cell subsets and serum immunoglobulin levels between WT and $L r r k 2^{-/-}$mice}

To define the role of LRRK2 in SLE development, Lrrk2 $2^{-/-}$mice were introduced in our study. With abrogated LRRK2 expression in Lrrk2 $^{-/-}$mice (Additional file 1: Figure S2), the effects of LRRK2 on humoral immunity at a steady state were determined first. There was no significant difference in the cellularity of total splenocytes between WT and Lrrk2 $^{-/-}$ mice (data not shown). We further examined B cell subsets in the spleens of WT and Lrrk2 ${ }^{-/-}$mice. After leaving bone marrow, immature $B$ cells embark the spleens and differentiate into mature B cells through a transition stage [26]. Comparable percentages of transition (TS) B cells $\left(\mathrm{B} 220^{+} \mathrm{CD}^{+} 3^{+}\right)$were present in the spleens of WT and Lrrk2 $2^{-/-}$mice. Disruption of Lrrk2 gene had no effect on the development of mature B cells $\left(\mathrm{B} 220^{+} \mathrm{CD}^{-} 3^{-}\right.$) and follicular (FO) B cells $\left(\mathrm{B} 220^{+} \mathrm{CD}^{-} 3^{-} \mathrm{CD} 23^{+} \mathrm{IgM}^{\text {low/-}}\right)$, two subpopulations of peripheral B cells either (Fig. $3 \mathrm{a}-\mathrm{C}$ ). A fraction of bone marrow $B$ cell subset migrates to the marginal zone of the spleens constituting marginal zone (MZ) B cells $\left(\mathrm{B} 220^{+} \mathrm{CD} 93^{-} \mathrm{CD} 23^{-} \mathrm{IgM}^{+}\right)$. This population represents long-lived $\mathrm{B}$ cells with the ability to respond to T-independent antigens from blood-borne pathogens [27]. Similarly, no difference in MZB cells was observed between WT and $\mathrm{Lrrk}^{-/-}$mice either (Fig. 3a, c). Therefore, mice lack of LRRK2 displays no impairment in B cell development.

Simultaneously, we also measured the basal levels of IgG and IgM in the serum by ELISA. It was found that 

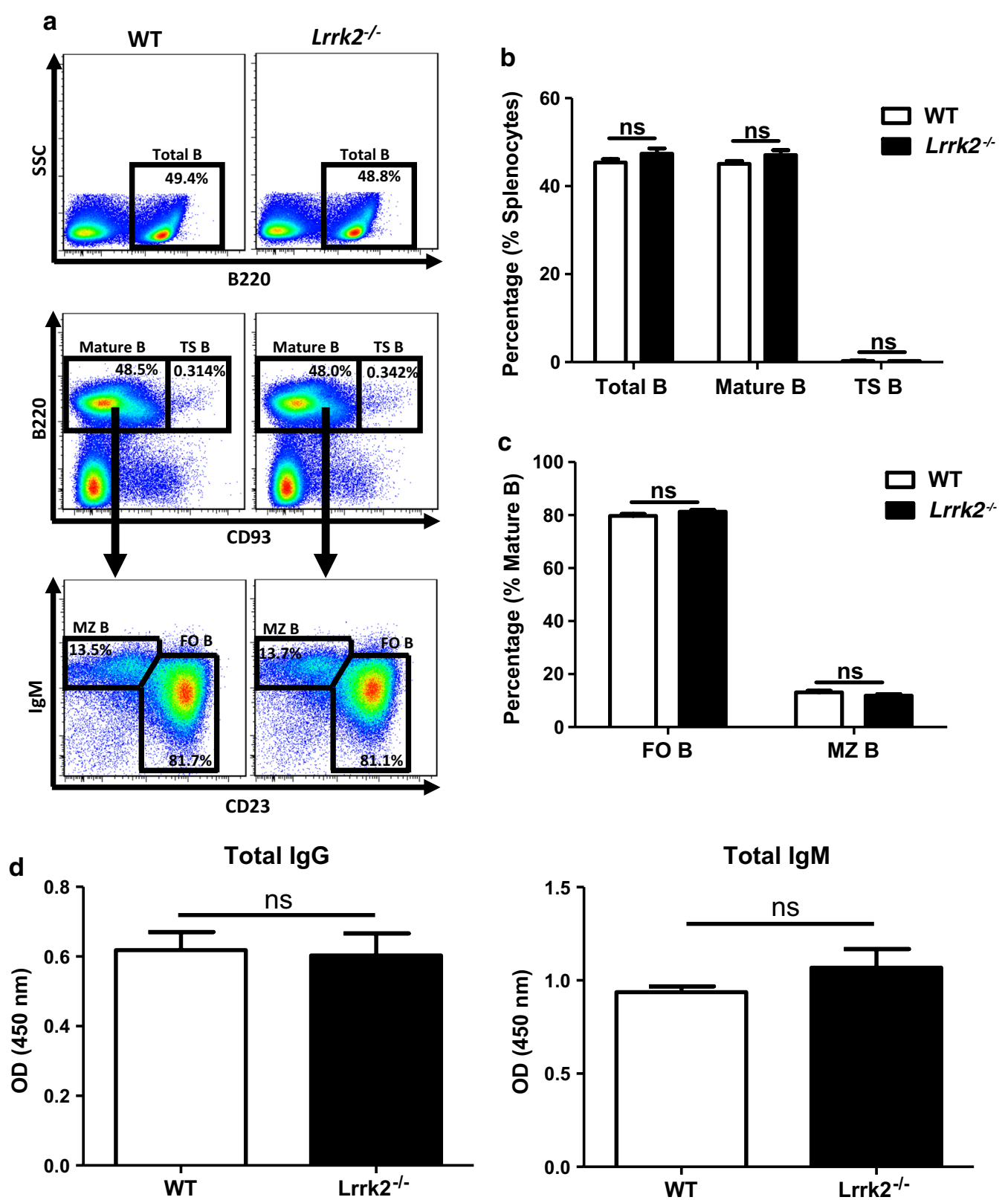

Fig. 3 Comparable B cell subsets and serum immunoglobulin levels between WT and Lrrk ${ }^{-1-}$ Mice. a Splenocytes were collected from WT and Lrrk2 $2^{-/-}$mice. B cell subsets in the periphery were analyzed by flow cytometry, including total B cells (B220+ lived cells, upper), mature B cells $\left(\mathrm{B} 220^{+} \mathrm{CD} 93^{-}\right.$, middle), transition (TS) B cells (B220+C CD93 ${ }^{+}$, middle), marginal zone (MZ) B cells (B220+ ${ }^{+} \mathrm{CD}_{3}{ }^{-} \mathrm{CD}_{23}{ }^{-} \mathrm{IgM}{ }^{+}$, low), and follicular (FO) B cells (B220+CD93-CD23+ ${ }^{+} \mathrm{gM}^{\text {low/- }}$, low). b Statistic percentages of total B cells, mature B cells and TS B cells in the spleens of WT and Lrrk2 ${ }^{-/-}$ mice. $\mathbf{c}$ Statistic percentages of MZ and FO B cells in the spleens of WT and $L r r k 2^{-/-}$mice. $\mathbf{d}$ Sera from WT and $L r r k 2^{-/-}$mice were subjected to the determination of total IgG and IgM by ELISA as described in "Methods". $\mathrm{OD}_{450}$ value was detected to represent IgG (left) and lgM (right) levels in the serum of the mice. Each group had at least six mice for the analysis. ns not significant

IgG and IgM levels were identical between $L r r k 2^{-/-}$mice and WT littermates (Fig. 3d), further supporting the fact that no commitment of LRRK2 occurs during B cell development and differentiation at the steady state.

\section{LRRK2 deficiency reduces antibody production} in pristane-treated mice

Pristane-induced lupus-like mouse model represents one of the models recapitulating key immunologic and clinical features of human SLE, including high percentages 
of IFN- $\alpha$ producing pDCs, production of autoantibodies against small nuclear ribonucleoproteins (snRNPs) and dsDNA, as well as the development of IC mediated glomerulonephritis [28]. Although $\operatorname{Lrrk}^{-/-}$mice exhibit normal B cell development and function at the steady state, whether pristane treatment induces different lupus-like phenotypes is worthy of investigation (Fig. 4a). It was found that there was an increased survival rate of

a

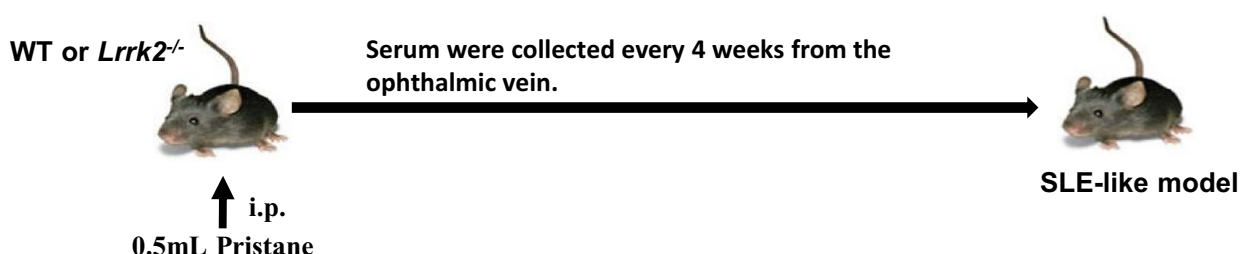

0.5mL Pristane

b

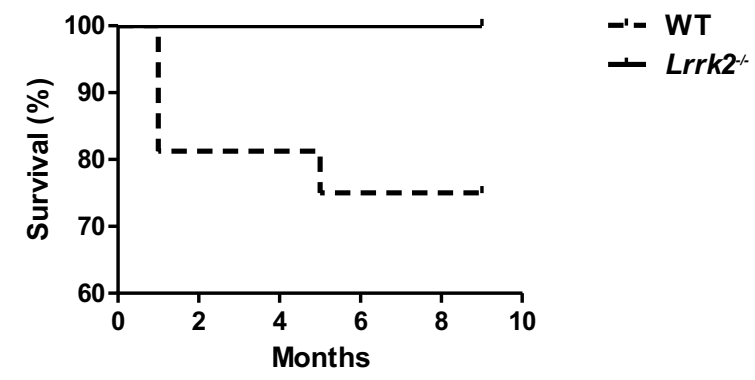

C

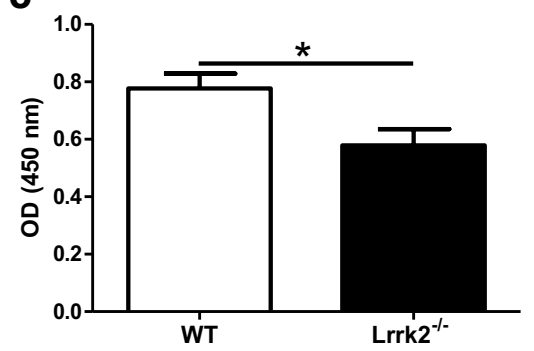

e

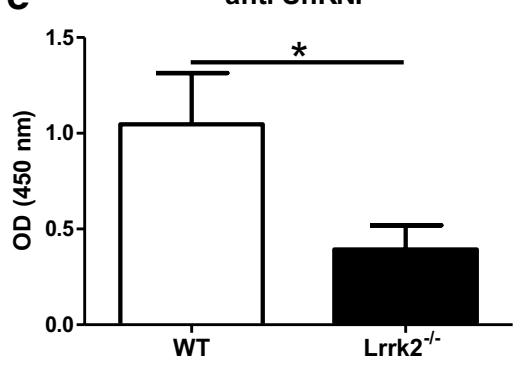

d

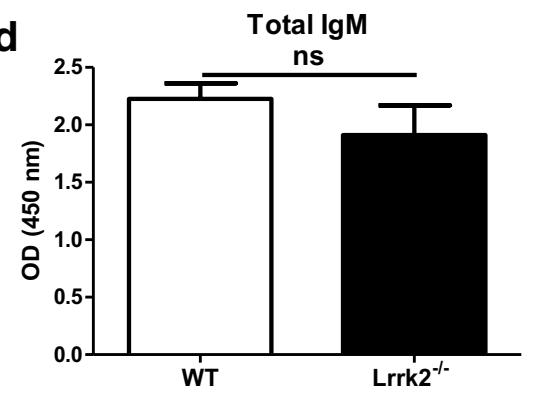

f

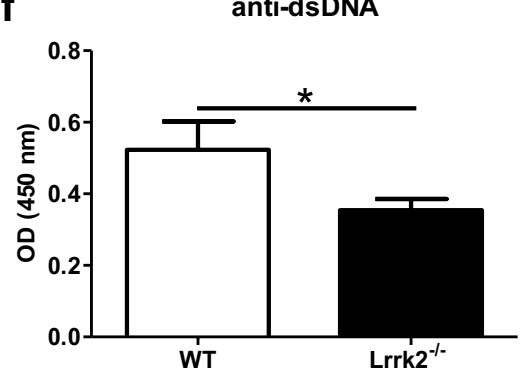

Fig. 4 LRRK2 deficiency attenuates pristane-induced antibody production in the mice. a Scheme of experiment design. WT and $L r r k 2^{-/-}$mice were intraperitoneally injected with $0.5 \mathrm{~mL}$ pristane. Sera were collected monthly for IgG and IgM determination. Mice were sacrificed 9 months later. b Survival rate of WT and $L r r k 2^{-/-}$mice after pristane injection. c-f Antibody levels in the sera of WT and $L r r k 2^{-/-}$mice were assayed by ELISA at 9 months after pristane injection, including total $\lg G(\mathbf{c})$, total $\operatorname{lgM}(\mathbf{d})$, anti-SnRNP antibody (e) and anti-dsDNA antibody (f). Each group had at least six mice for antibody analysis. ${ }^{*} p<0.05$; ns not significant 
Lrrk2 ${ }^{-/-}$mice in comparison to WT mice after pristane injection (Fig. 4b). We next compared the antibody production between WT and Lrrk2 $2^{-/-}$mice. The levels of serum total IgG and autoantibodies collected monthly after the injection increased gradually both in WT and LRRK2 knockout mice (Additional file 1: Figure S3). At 9 months after pristane injection, however, total IgG level was significantly lower in $L r r k 2^{-/-}$mice than that in WT mice (Fig. 4c) whereas total IgM was comparable between two types of mice (Fig. 4d). When comparing autoantibody levels between WT and Lrrk2 $2^{-/}$mice, it was obvious that both anti-snRNP and anti-dsDNA autoantibodies were lower in $L r r k 2^{-/-}$mice than WT counterparts where anti-snRNP was more dramatic (Fig. 4e, f).

\section{LRRK2 deficiency attenuates pristane-induced lupus-like pathology in mice}

Considering that high LRRK2 expression in B cells from SLE patients was strongly correlated with low levels of serum $\mathrm{C} 3$ and $\mathrm{C4}$, two indicators related to renal injury during SLE pathogenesis [29], whether decreased total IgG and autoantibodies levels in $L r r k 2^{-/-}$mice upon pristane treatment led to less kidney pathology was further investigated at 9 months post pristane treatment. It was revealed that the severity of nephritis was ameliorated in $L r r k 2^{-/-}$mice reflected by less glomeruli represented by less endocapillary or extracapillary proliferative lesions and sclerotic lesions (Fig. 5a, b). The deposit of IgG in the kidneys of Lrrk2 $2^{-/-}$mice was reduced significantly as well (Fig. 5c, d). Altogether, our results indicate
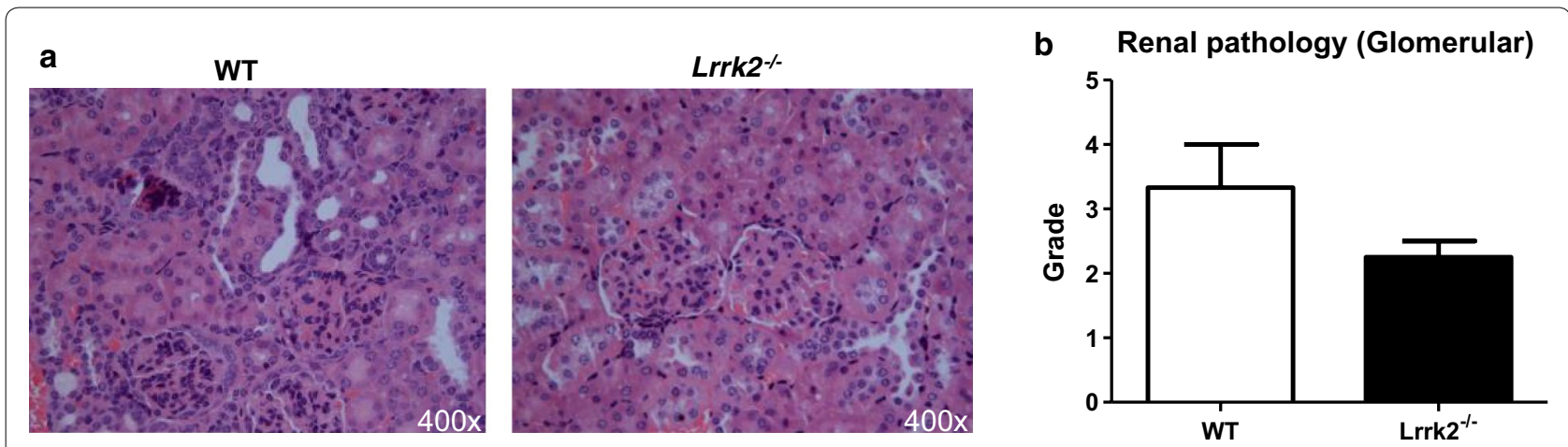

C
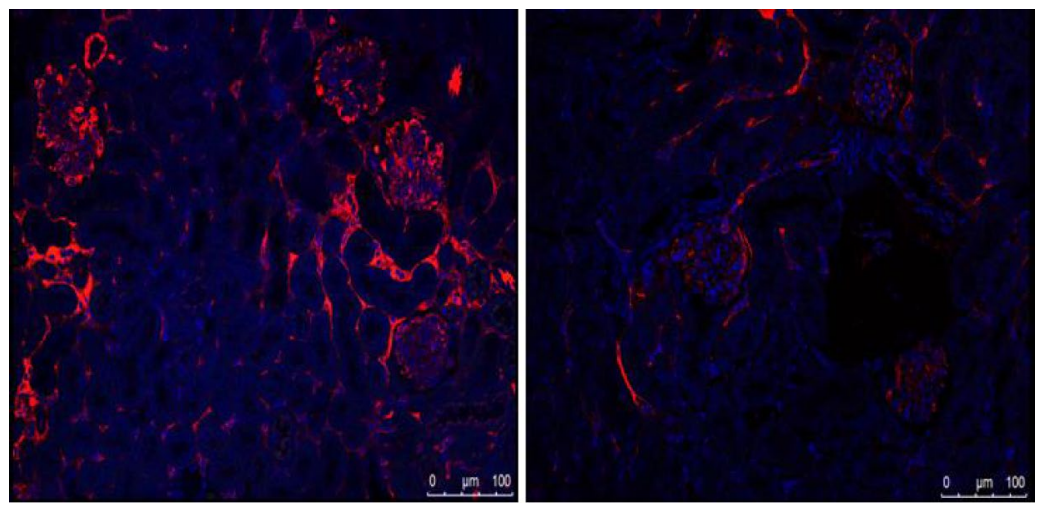

d

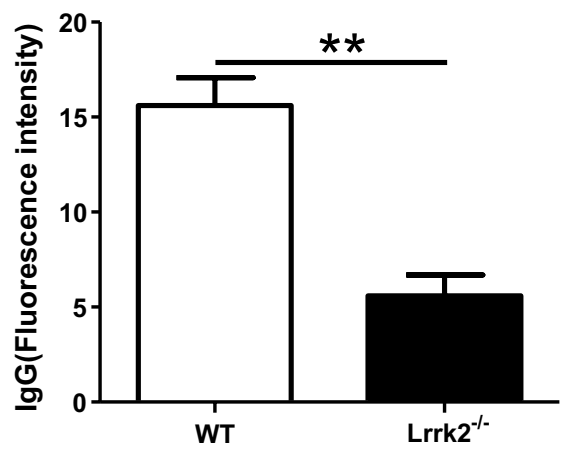

DAPI

$\lg \mathbf{G}$

Fig. 5 Pathological properties in mouse kidneys after pristane treatment. a The kidney pathology was investigated at 9 months post pristane treatment. Glomerular injury with mesangial expansion and lymphocyte infiltration in the kidneys of WT and $L \mathrm{rrk} 2^{-/-}$mice was determined by H\&E staining $(400 \times)$. b Semiquantitative analysis of glomerulonephritis scores. The scores were obtained based on the number of the glomeruli with endocapillary proliferation, extracapillary proliferation (crescents), the damage grade was evaluated as described in "Methods". c Representatives of glomerular IgG deposition in the kidneys of WT and $\mathrm{rrrk}^{-1-}$ mice (200X). d The mean fluorescence intensity (MFI) of IgG within glomeruli was determined by ImageJ software. Both statistics were based on at least five randomly selected images of each mouse. Each group had at least six mice for pathological analysis. ${ }^{* *} \mathrm{p}<0.01$ 
that LRRK2 deficiency exhibits the protection against pristane-induced lupus-like pathology, partially owing to less production of total IgG and autoantibodies.

\section{Decreased percentages of germinal center B cells} and plasma cells in $\mathbf{L r k} \mathbf{2}^{-/-}$mice after pristane injection

To explore the mechanisms of less production of total IgG and autoantibody in Lrrk2 $2^{-/-}$mice, we further analyzed B cell terminal differentiation closely related to antibody production after pristane injection. Germinal center (GC) B cells and plasma cells are two key subpopulations at the terminal stage of $B$ cell differentiation occurring in GCs responsible for antibody production upon antigen stimulation [30]. At present, it is not clear how pristane induces the release of autoantigens and triggers $B$ cell differentiation. Nevertheless, we were inclined to compare the percentages of GC B cells and plasma cells between WT and $L r r k 2^{-/-}$mice at 9 months post pristane treatment. Consistent with the antibody results, $\mathrm{Lrrk}^{-/-}$mice displayed the remarkable decrease in GC B cells and plasma cells in the spleens upon pristane treatment (Fig. 6a, b). Therefore, pristane-induced
LRRK2 deficient mice exhibit impaired B cell terminal differentiation, which is probably responsible for the attenuated Ig production and subsequent less IC deposit in the kidneys.

\section{Discussion}

SLE is an autoimmune disease that manifests the generation of autoantibodies and the deposition of ICs in certain tissues and organs. Autoreactive B cells are a key mediator for disease progression. While their hyperactivation is supposed to be exaggerated by type I interferon [31] or TLR7/9 signaling [32], B cell differentiation in SLE is also programmed by multiple factors $[1,33]$. In this study, the role of LRRK2 as a novel potential regulator in the pathogenesis of SLE was suggested based on the correlation of abnormal LRRK2 expression in B cells with disease severity of SLE, and further validated in a pristane-induced lupus-prone model. The engagement of LRRK2 in the pathogenesis of SLE was probably through affecting $B$ cell terminal differentiation and the production of pathological antibodies. a

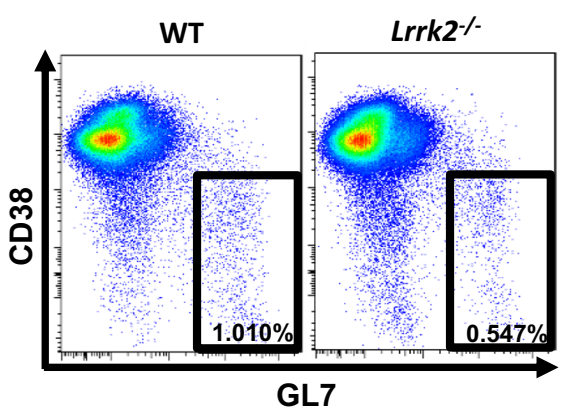

b Gate on $\mathrm{CD}_{19}{ }^{+} \mathrm{B220}+\mathrm{B}$ cells

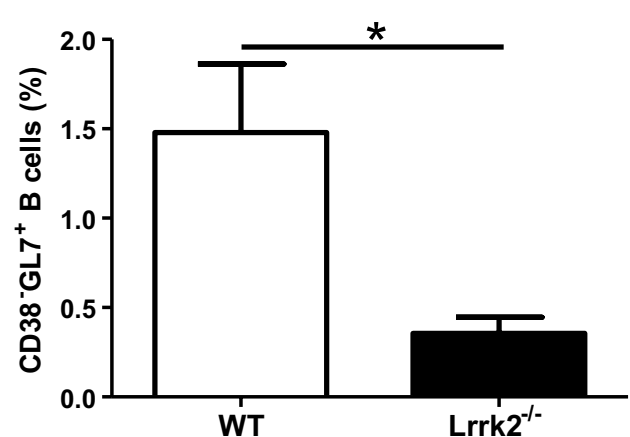

\section{Gate on splenocytes}

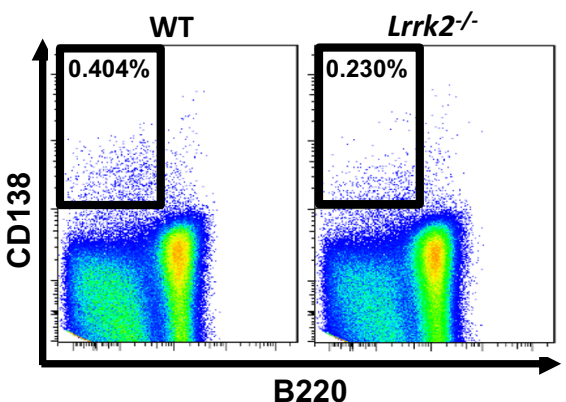

Gate on splenocytes

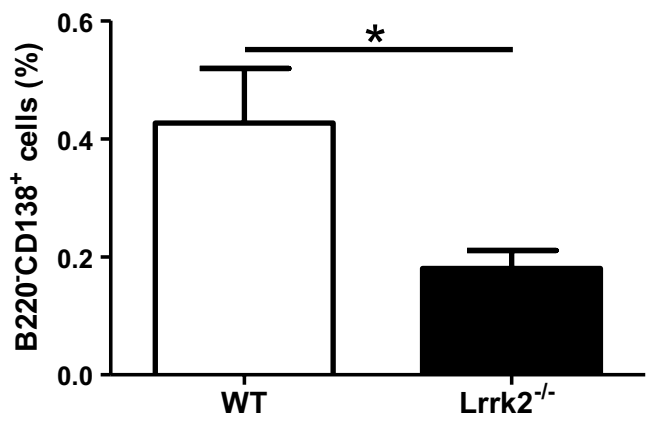

Fig. 6 Decreased percentages of GC B cells and plasma cells in $L r r k 2^{-/-}$mice after pristane injection. a Splenocytes were collected from the spleens of WT and $L r r k 2^{-/-}$mice at 9 months post pristane treatment. Flow cytometric analysis were performed to determine the percentages of

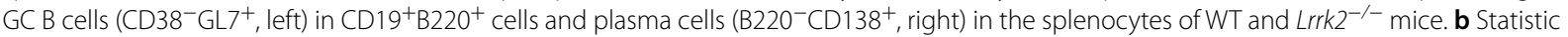
results of the percentages of GC B (left) and plasma cells (right) in the spleens of WT and $L r r k 2^{-/-}$mice after pristane injection for 9 months. Each group had at least six mice for analysis. * $\mathrm{p}<0.05$ 
LRRK2 is a kinase protein with its variants associated with multiple diseases including Parkinson's disease (PD) [12], IBD [15, 16] and cancer [17]. A recent report also suggested that LRRK2 variant was associated with SLE [9]. Interestingly, LRRK2 was previously reported to be highly expressed in B cells [21]. We extend this notion by showing abnormally high expression of LRRK2 in B cells from SLE patients. Furthermore, we demonstrated that abnormal expression of LRRK2 in B cells was correlated with SLEDAI, C3 and C4 levels which were key standards for SLE diagnosis. These data also suggest that LRRK2 may have a critical role in B cell immunity. Surprisingly, we couldn't observe any effects of LRRK2 on B cell development and humoral immune response at the steady state in mice, which is inconsistent with a previous study [34]. The reason for this discrepancy is still unknown. One possible reason may be the environment difference which may lead to microbiota change. It's worth to address this point in the future.

LRRK2 is highly expressed in B cells, monocytes, and dendritic cells suggesting its potential function in immune system [11]. LRRK2 expression is induced by interferon- $\gamma$ (IFN- $\gamma$ ) and lipopolysaccharide (LPS) which is important for its antimicrobial activities [11]. Its deficiency exacerbated colitis in mice treated with dextran sodium sulphate [16]. Mechanistic studies showed that LRRK2 was a potent negative regulator of the transcription factor NFAT [16]. Recently, LRRK2 was demonstrated to be required for lysozyme sorting in Paneth cells directed by commensal bacteria through a Nod2-LRRK2-Rab2a axis [18]. We recently reported LRRK2 was critical for NLRC4 inflammasome activation [20]. Collectively, these emerging data implies the possible roles of LRRK2 in immune modulation. While many of these studies suggest that LRRK2 is critical in innate immunity, its role in adaptive immunity is largely unknown. Here we found that abnormal LRRK2 expression was closely associated with SLE severity. LRRK2 promoted B cell maturation and hyper-activation, increased autoantibody production and aggravated pristane-induced lupus in a SLE animal model, thus implicating LRRK2 as a new regulator of $B$ cell function and humoral immunity. The underlying mechanisms warrant further investigation.

$L R R K 2$ gene polymorphism is originally dedicated to PD incidence, a disease characterized as a neurodegenerative disorder with the loss of midbrain dopaminergic neurons [13]. However, more and more reports describe the occurrence of inflammation and the elevation of inflammatory cytokines in the brains and cerebrospinal, alternatively supporting the roles of LRRK2 in immune regulation and making it a potential target for disease control. Several favorable candidate inhibitors have been developed for the experimental control of PD based on the kinase activity of LRRK2, such as LRRK2-IN-1 [35], CZC-54252 and CZC-25146 [36], GSK2578215A [37] etc. The LRRK2 inhibitor, PF-06447475, effectively attenuates $\alpha$-synuclein-induced dopaminergic neurodegeneration [38]. In addition, LRRK2 inhibitor treatment appears to promote the ubiquitination of LRRK2 and consequently accelerate its proteasomal degradation [39]. Considering the increase of LRRK2 expression in B cells and the involvement in the pathogenesis of SLE, whether the existing LRRK2 inhibitor can specifically retard the B cell activation and differentiation for disease remission is worthy of further exploration.

\section{Conclusion}

In summary, our study present here provides the direct evidence on the involvement of LRRK2 in the pathogenesis of SLE in mice. This is probably due to its roles in regulating $B$ cell terminal differentiation, which in turn affects the antibody production and disease progression. Considering the exclusively high expression of LRRK2 in B cells from SLE patients, the pathological roles of LRRK2 in human SLE might be similar to a large extent. Once the underlying mechanisms are clarified, LRRK2 might become a novel target for developing new diagnostic and therapeutic strategy for the control of SLE.

\section{Additional file}

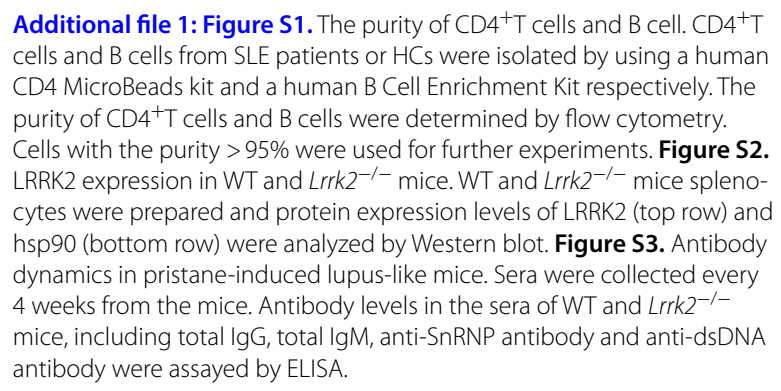
cells and B cells from SLE patients or HCs were isolated by using a human CD4 MicroBeads kit and a human B Cell Enrichment Kit respectively. The purity of $C D 4^{+} T$ cells and B cells were determined by flow cytometry. Cells with the purity $>95 \%$ were used for further experiments. Figure $\mathbf{S 2}$. LRRK2 expression in WT and Lrrk2 ${ }^{-/-}$mice. WT and Lrrk2 ${ }^{-1-}$ mice splenocytes were prepared and protein expression levels of LRRK2 (top row) and hsp90 (bottom row) were analyzed by Western blot. Figure S3. Antibody dynamics in pristane-induced lupus-like mice. Sera were collected every 4 weeks from the mice. Antibody levels in the sera of WT and $L r r k 2^{-/-}$ mice, including total lgG, total IgM, anti-SnRNP antibody and anti-dsDNA antibody were assayed by ELISA.

\section{Abbreviations}

SLE: systemic lupus erythematosus; LRRK2: leucine-rich repeat kinase 2; SLEDAl: systemic lupus erythematosus disease activity index; pDCs: plasmacytoid dendritic cells; GWAS: genome-wide associated analysis; IBD: inflammatory bowel disease; PBMCs: peripheral blood mononuclear cells; CVID: common variable immunodeficiency; snRNPs: small nuclear ribonucleoproteins; GC: germinal center; PD: Parkinson's disease.

\section{Authors' contributions}

YW and ZZK initiated and designed the study. MYZ, CCY, SL and XNL performed the animal experiments. JC designed and preformed the histological study. YYC, SJW and PJ performed the human study. MP collected the clinical samples. YW, ZZK, MYZ and CCY performed the data analysis and 
interpretation. YW, ZZK and MYZ wrote the manuscript. All authors read and approved the final manuscript.

\begin{abstract}
Author details
${ }^{1}$ Shanghai Institute of Immunology, Department of Immunology and Microbiology, Shanghai Jiao Tong University School of Medicine, Shanghai 200025, China. ${ }^{2}$ Department of Dermatology, Rui Jin Hospital, Shanghai Jiao Tong University School of Medicine, Shanghai 200025, China. ${ }^{3}$ Department of Inflammation and Immunity, Cleveland Clinic, Cleveland, OH 44195, USA. ${ }^{4}$ Department of Molecular Medicine, Cleveland Clinic Lerner College of Medicine, Case Western Reserve University, Cleveland, OH 44106, USA. ${ }^{5}$ Department of Clinical Laboratory, The First Affiliated Hospital of Zhengzhou University, Zhengzhou 450052, China. ${ }^{6}$ Department of Pathology, Shanghai Jiao Tong University School of Medicine, Shanghai 200025, China.
\end{abstract}

\section{Acknowledgements}

We gratefully appreciated Dr. Chuan-xin Huang and Prof Bing Su for their kind suggestions on this study. We also thanked Wenwen Liu and Xujiao Feng (Shanghai Institute of Immunology, Institute of Medical Sciences, Shanghai Jiao Tong University School of Medicine) for their assistance in flow cytometry.

\section{Competing interests}

The authors declare that they have no competing interests.

\section{Availability of data and materials}

All data generated or analyzed during this study were included in this published article and its additional file.

\section{Consent for publication}

Each person provided signed informed consent for publication of the results of the study.

\section{Ethics approval and consent to participate}

Informed consent was obtained from all participants included in the study. The animal study was approved by the Scientific Investigation Board of Shanghai Jiao Tong University School of Medicine.

\section{Funding}

This work was supported by the grants from the National Science Foundation of China (31370884) and Shanghai Academic Research Leader Project (18XD1403300).

\section{Publisher's Note}

Springer Nature remains neutral with regard to jurisdictional claims in published maps and institutional affiliations.

Received: 25 September 2018 Accepted: 11 January 2019

Published online: 22 January 2019

\section{References}

1. Tsokos GC. Systemic lupus erythematosus. N Engl J Med. 2011;365:2110-21.

2. Dorner T, Giesecke C, Lipsky PE. Mechanisms of B cell autoimmunity in SLE. Arthritis Res Ther. 2011;13:243.

3. Beckwith $\mathrm{H}$, Lightstone L. Rituximab in systemic lupus erythematosus and lupus nephritis. Nephron Clin Pract. 2014;128:250-4.

4. Martínez R, Muñoz A, Velloso ML, Rodríguez Montero S, Belmonte-López MA, Marenco JL. Rituximab is effective in the treatment of nephritis in lupus patients, refractory to conventional immunosuppressive therapy. J Transl Med. 2010;8:P71.

5. Malkiel S, Barlev AN, Atisha-Fregoso Y, Suurmond J, Diamond B. Plasma cell differentiation pathways in systemic lupus erythematosus. Front Immunol. 2018;9:427.

6. Dolff S, Bijl M, Huitema MG, Limburg PC, Kallenberg CG, Abdulahad WH. Disturbed Th1, Th2, Th17 and T(reg) balance in patients with systemic lupus erythematosus. Clin Immunol. 2011;141:197-204.
7. Alculumbre S, Raieli S, Hoffmann C, Chelbi R, Danlos FX, Soumelis V. Plasmacytoid pre-dendritic cells ( $(\mathrm{DCC})$ : from molecular pathways to function and disease association. Semin Cell Dev Biol. 2018.

8. Cui Y, Sheng Y, Zhang X. Genetic susceptibility to SLE: recent progress from GWAS. J Autoimmun. 2013:41:25-33.

9. Zhang YM, Zhou XJ, Cheng FJ, Qi YY, Hou P, Zhao MH, Zhang H. Autophagy-related gene LRRK2 is likely a susceptibility gene for systemic lupus erythematosus in northern Han Chinese. Oncotarget. 2017:8:13754-61.

10. Zimprich A, Biskup S, Leitner P, Lichtner P, Farrer M, Lincoln S, Kachergus J, Hulihan M, Uitti RJ, Calne DB, et al. Mutations in LRRK2 cause autosomal-dominant parkinsonism with pleomorphic pathology. Neuron. 2004:44:601-7.

11. Gardet A, Benita Y, Li C, Sands BE, Ballester I, Stevens C, Korzenik JR, Rioux JD, Daly MJ, Xavier RJ, Podolsky DK. LRRK2 is involved in the IFN-gamma response and host response to pathogens. J Immunol. 2010;185:5577-85.

12. Rideout HJ, Stefanis L. The neurobiology of LRRK 2 and its role in the pathogenesis of Parkinson's disease. Neurochem Res. 2014;39:576-92.

13. Juarez-Flores DL, Gonzalez-Casacuberta I, Ezquerra M, Bano M, CarmonaPontaque F, Catalan-Garcia M, Guitart-Mampel M, Rivero JJ, Tobias E, Milisenda JC, et al. Exhaustion of mitochondrial and autophagic reserve may contribute to the development of LRRK2 (G2019S)-Parkinson's disease. J Transl Med. 2018;16:160.

14. Wang D, Xu L, Lv L, Su LY, Fan Y, Zhang DF, Bi R, Yu D, Zhang W, Li XA, et al. Association of the LRRK2 genetic polymorphisms with leprosy in Han Chinese from Southwest China. Genes Immun. 2015;16:112-9.

15. Liu Z, Lenardo MJ. The role of LRRK2 in inflammatory bowel disease. Cell Res. 2012;22:1092-4.

16. Liu Z, Lee J, Krummey S, Lu W, Cai H, Lenardo MJ. The kinase LRRK2 is a regulator of the transcription factor NFAT that modulates the severity of inflammatory bowel disease. Nat Immunol. 2011;12:1063-70.

17. Ruiz-Martinez J, de la Riva P, Rodriguez-Oroz MC, Mondragon Rezola E, Bergareche A, Gorostidi A, Gago B, Estanga A, Larranaga N, Sarasqueta C, et al. Prevalence of cancer in Parkinson's disease related to R1441G and G2019S mutations in LRRK2. Mov Disord. 2014;29:750-5.

18. Zhang Q, Pan Y, Yan R, Zeng B, Wang H, Zhang X, Li W, Wei H, Liu Z. Commensal bacteria direct selective cargo sorting to promote symbiosis. Nat Immunol. 2015;16:918-26.

19. Toledo Pinto TG, Batista-Silva LR, Medeiros RCA, Lara FA, Moraes MO. Type I interferons, autophagy and host metabolism in leprosy. Front Immunol. 2018:9:806.

20. Liu W, Liu X, Li Y, Zhao J, Liu Z, Hu Z, Wang Y, Yao Y, Miller AW, Su B, et al. LRRK2 promotes the activation of NLRC4 inflammasome during Salmonella Typhimurium infection. J Exp Med. 2017;214:3051-66.

21. Maekawa T, Kubo M, Yokoyama I, Ohta E, Obata F. Age-dependent and cell-population-restricted LRRK2 expression in normal mouse spleen. Biochem Biophys Res Commun. 2010;392:431-5.

22. Warnatz K, Wehr C, Drager R, Schmidt S, Eibel H, Schlesier M, Peter HH. Expansion of CD19(hi)CD21(lo/neg) B cells in common variable immunodeficiency (CVID) patients with autoimmune cytopenia. Immunobiology. 2002;206:502-13.

23. Nicholas MW, Dooley MA, Hogan SL, Anolik J, Looney J, Sanz I, Clarke SH. A novel subset of memory B cells is enriched in autoreactivity and correlates with adverse outcomes in SLE. Clin Immunol. 2008;126:189-201.

24. Liu Z, Zeng W, Huang $X$, Wang S, Zheng J, Pan M, Wang Y. Peripheral CD19(hi) B cells exhibit activated phenotype and functionality in promoting $\lg G$ and $\lg M$ production in human autoimmune diseases. Sci Rep. 2017;7:13921.

25. Chen M, Daha MR, Kallenberg CG. The complement system in systemic autoimmune disease. J Autoimmun. 2010;34:J276-86.

26. Wang Y, Horvath O, Hamm-Baarke A, Richelme M, Gregoire C, Guinamard R, Horejsi V, Angelisova P, Spicka J, Schraven B, et al. Single and combined deletions of the NTAL/LAB and LAT adaptors minimally affect B-cell development and function. Mol Cell Biol. 2005:25:4455-65.

27. Lopes-Carvalho T, Kearney JF. Development and selection of marginal zone B cells. Immunol Rev. 2004;197:192-205.

28. Reeves WH, Lee PY, Weinstein JS, Satoh M, Lu L. Induction of autoimmunity by pristane and other naturally occurring hydrocarbons. Trends Immunol. 2009;30:455-64.

29. Garin EH, Donnelly WH, Shulman ST, Fernandez R, Finton C, Williams RL, Richard GA. The significance of serial measurements of serum 
complement C3 and C4 components and DNA binding capacity in patients with lupus nephritis. Clin Nephrol. 1979;12:148-55.

30. Shlomchik MJ, Weisel F. Germinal center selection and the development of memory B and plasma cells. Immunol Rev. 2012;247:52-63.

31. Niewold TB, Hua J, Lehman TJ, Harley JB, Crow MK. High serum IFN-alpha activity is a heritable risk factor for systemic lupus erythematosus. Genes Immun. 2007:8:492-502.

32. Santiago-Raber ML, Baudino L, Izui S. Emerging roles of TLR7 and TLR9 in murine SLE. J Autoimmun. 2009:33:231-8.

33. Min HK, Kim SM, Park JS, Byun JK, Lee J, Kwok SK, Park YW, Cho ML, Park SH. Fn14-Fc suppresses germinal center formation and pathogenic B cells in a lupus mouse model via inhibition of the TWEAK/Fn14 pathway. J Transl Med. 2016;14:98.

34. Kubo M, Nagashima R, Ohta E, Maekawa T, Isobe Y, Kurihara M, Eshima K, Iwabuchi K, Sasaoka T, Azuma S, et al. Leucine-rich repeat kinase 2 is a regulator of $B$ cell function, affecting homeostasis, $B C R$ signaling, IgA production, and TI antigen responses. J Neuroimmunol. 2016;292:1-8.
35. Koshibu K, van Asperen J, Gerets H, Garcia-Ladona J, Lorthioir O, Courade JP. Alternative to LRRK2-IN-1 for pharmacological studies of Parkinson's disease. Pharmacology. 2015;96:240-7.

36. Ramsden N, Perrin J, Ren Z, Lee BD, Zinn N, Dawson VL, Tam D, Bova $M$, Lang M, Drewes $G$, et al. Chemoproteomics-based design of potent LRRK2-selective lead compounds that attenuate Parkinson's diseaserelated toxicity in human neurons. ACS Chem Biol. 2011;6:1021-8.

37. Reith AD, Bamborough $P$, Jandu K, Andreotti D, Mensah L, Dossang $P$, Choi HG, Deng X, Zhang J, Alessi DR, Gray NS. GSK2578215A; a potent and highly selective 2-arylmethyloxy-5-substitutent- $N$-arylbenzamide LRRK2 kinase inhibitor. Bioorg Med Chem Lett. 2012;22:5625-9.

38. Daher JP, Abdelmotilib HA, Hu X, Volpicelli-Daley LA, Moehle MS, Fraser KB, Needle E, Chen Y, Steyn SJ, Galatsis P, et al. Leucine-rich repeat kinase 2 (LRRK2) pharmacological inhibition abates alpha-synuclein geneinduced neurodegeneration. J Biol Chem. 2015;290:19433-44.

39. Zhao J, Molitor TP, Langston JW, Nichols RJ. LRRK2 dephosphorylation increases its ubiquitination. Biochem J. 2015;469:107-20.
Ready to submit your research? Choose BMC and benefit from:

- fast, convenient online submission

- thorough peer review by experienced researchers in your field

- rapid publication on acceptance

- support for research data, including large and complex data types

- gold Open Access which fosters wider collaboration and increased citations

- maximum visibility for your research: over $100 \mathrm{M}$ website views per year

At BMC, research is always in progress.

Learn more biomedcentral.com/submissions 